\title{
Balão Térmico para Ablação Endometrial: Resultados Preliminares
}

\author{
Endometrial Ablation Using a Thermal Balloon: Preliminary Results
}

Francesco Viscomi ${ }^{1}$, João Alfredo Martins ${ }^{1}$, Marilice Pastore ${ }^{1}$, Rogério Dias ${ }^{2}$, Laurival DeLuca ${ }^{2}$

\begin{abstract}
RESUMO
Objetivos: os autores mostram a experiência preliminar com o uso do balão térmico para ablação do endométrio em pacientes com queixas de menorragia.

Pacientes e Métodos: foram submetidas a este procedimento 20 pacientes. Após exame pélvico completo e ultra-sonografia endovaginal, todas as pacientes foram submetidas à histeroscopia diagnóstica com biópsia de endométrio para excluir causas de malignidade. Das 20 pacientes que se submeteram ao tratamento com balão térmico, 16 foram submetidas em regime ambulatorial com anestesia local. O procedimento teve duração de 8 minutos e 30 segundos. Resultados: duas das 20 pacientes mostraram-se insatisfeitas, mantendo o quadro hemorrágico inalterado, e 18 pacientes referiram melhora da sintomatologia. Não houve complicações do procedimento.

Conclusões: o uso do balão térmico para ablação endometrial mostrou-se seguro e eficaz para o tratamento da menorragia de causa benigna.
\end{abstract}

PALAVRAS-CHAVE: Sangramento uterino anormal. Endometrio: ablação. Balão térmico. Histeroscopia.

\section{Introdução}

A mudança do perfil reprodutivo da mulher nos últimos anos tem originado uma maior freqüência de queixas relacionadas ao sangramento uterino menstrual. Virtualmente cada mulher, em algum momento da vida, pode experimentar episódio de sangramento menstrual abundante ou menorragia ${ }^{1}$.

De acordo com a Organização Mundial de Saúde (OMS), em relatos de diferentes países num total de 5.322 mulheres a prevalência de menorragia foi de $19 \%{ }^{2}$. A menorragia é um sintoma que, além de debilitar o organismo da mulher, por vezes pode impedi-la de exercer suas atividades profissionais.

A abordagem terapêutica da menorragia na ausência de malignidade é um dos problemas

${ }^{1}$ Women's Medical Center - São Paulo

${ }^{2}$ Departamento de Tocoginecologia da Faculdade de Medicina de Botucatu - UNESP

Correspondência: Francesco Antonio Viscomi

Av. Eusébio Matoso, 196 - Pinheiros

05423-000 - São Paulo - SP

Tel.: (11) 870-0577 mais freqüentes encontrados pelo ginecologista. O tratamento convencional resume-se numa abordagem clínica ou cirúrgica. As várias drogas utilizadas no tratamento da menorragia podem reduzir o fluxo menstrual em $25-80 \%{ }^{3}$. Entretanto, sua eficácia depende da continuação do uso da droga e habitualmente os sintomas retornam após interrupção do tratamento. Deve-se considerar também que algumas pacientes apresentam contra-indicação para tratamentos hormonais, assim como estes podem apresentar efeitos colaterais. Em relação ao tratamento cirúrgico, a curetagem e a histerectomia têm sido os dois procedimentos mais utilizados. A curetagem uterina tem um efeito temporário sobre a menorragia e o tratamento precisaria ser repetido várias vezes, além do que o seu efeito terapêutico está limitado aos primeiros ciclos menstruais após o procedimento ${ }^{4}$.

Embora a histerectomia leve à cura da menorragia, deve-se considerar sempre a morbidade do procedimento. No passado a morbidade era de $25-50 \%$, representada principalmente por infecção intra-abdominal, infecção na incisão e hemorragia $^{5}$. Atualmente a morbidade é significativamente 
menor, com taxas ao redor de $3 \% \%^{6}$. A mortalidade com a histerectomia, porém, apresenta taxas que variam de 6 a 11 por 10.000 procedimentos $^{6}$.

Recentemente algumas alternativas têm sido desenvolvidas com o objetivo de melhorar a eficácia da curetagem uterina e diminuir a necessidade de histerectomia. Várias técnicas ablativas sobre o endométrio vêm sendo desenvolvidas nos casos de sangramento uterino anormal de causa benigna. Estes métodos incluem o uso de neodímio Yag (Nd : Yag) laser de contacto e não-contacto ${ }^{7,8}$, a eletrocirurgia usando alça elétrica e ressectoscópio ${ }^{9,10}$, ou a alça elétrica em roller-ball ${ }^{11}$. Técnicas ablativas sem a visualização direta têm sido utilizadas para destruir o endométrio, incluindo criocirurgia ${ }^{12}$ e diatermia por micro-ondas ${ }^{13}$, porém estas técnicas têm sido utilizadas em pequenos grupos e ainda em caráter experimental.

A ablação térmica com uso de balão consiste na introdução de um cateter dotado de um balão de látex na extremidade, que introduzido na cavidade uterina é preenchido com solução estéril de dextrose $5 \%$. O tratamento resulta na coagulação uniforme do endométrio por calor por meio do líquido existente no balão. Esta avaliação preliminar faz parte de um estudo multicêntrico que vem sendo realizado em serviços da Europa, Estados Unidos e México.

\section{Pacientes e Métodos}

A ablação do endométrio com uso do balão térmico endo-uterino foi realizada no período de junho de 1996 a junho de 1997 em 20 pacientes que apresentavam queixa de menorragia. A idade das pacientes variou de 34 a 54 anos. Em relação a métodos contraceptivos, oito pacientes referiam laqueadura tubária prévia, três utilizam contraceptivos orais, e os parceiros de duas delas eram vasectomizados, sete utilizavam preservativos. Das 20 pacientes, 6 apresentavam anemia. Todas as pacientes foram submetidas a exame ginecológico completo, com colpocitologia oncótica, ultra-sonografia endovaginal e histeroscopia diagnóstica com biópsia de endométrio.

A ultra-sonografia endovaginal revelou volume do útero variando de $75-110 \mathrm{~cm}^{3}$ e espessura endometrial em torno de $7 \mathrm{~mm}$. A histeroscopia diagnóstica afastou a presença de anormalidades da cavidade uterina nas pacientes selecionadas para aplicação do balão térmico endouterino. As análises histológicas das biópsias revelaram a presença de endométrio proliferativo em 9 casos; endométrio secretor em 7 casos e hi- perplasia simples sem atipias nos outros 4 casos. Todas as pacientes referiam tratamentos hormonal prévio sem sucesso. Nenhuma das pacientes foi submetida a uso de análogos de GnRH com objetivo de preparar o endométrio para aplicação do balão térmico.

Das 20 pacientes, em 4 o procedimento foi realizado em ambiente hospitalar sob anestesia geral. Duas destas pacientes receberam este tratamento em função da concomitância com outros procedimentos cirúrgicos (perineoplastia e polipectomia) e as outras duas devido a ansiedade relacionada com a anestesia local. As outras 16 pacientes foram submetidas à anestesia local. A anestesia local constou de aplicação no terço superior da vagina de um creme a base de lidocaina e prilocaina 30 minutos antes da infiltração dos ligamentos útero-sacros. A infiltração dos ligamentos útero-sacros foi realizada utilizando-se Xilocaina ${ }^{\circledR} 1,5 \%$ no volume de $5 \mathrm{ml}$, complementado por $2 \mathrm{ml}$ nos quadrantes do colo do útero.

Todas as pacientes foram submetidas à histeroscopia imediatamente após a aplicação do balão térmico, com objetivo de confirmar a ação do mesmo sobre as paredes uterinas.

O sistema de balão térmico consiste de um cateter que na sua extremidade distal está envolvido por um balão que contém um filamento, que é aquecido durante o procedimento. O cateter é conectado à unidade de controle, que indica a pressão, temperatura e tempo decorrido do procedimento. Se ocorrer falha na pressão ou temperatura, o aparelho é dotado de um sistema de segurança que automaticamente interrompe o procedimento.

O cateter é inserido na cavidade uterina e o balão é inflado até uma pressão em torno de 160 $\mathrm{mmHg}$ com solução estéril de dextrose $5 \%$. O elemento de aquecimento dentro do balão aumenta a temperatura do fluído para aproximadamente $87^{\circ} \mathrm{C}$ e mantém esta temperatura por 8 minutos. O endométrio é então coagulado por ação térmica até uma profundidade aproximada de $5 \mathrm{~mm}$. Quando o controle assinala que o procedimento foi realizado, soa um alarme indicando o momento para desinsuflar o balão e retirar o cateter.

As pacientes retornaram 40 dias após o procedimento e a cada 6 meses para avaliação da sintomatologia menstrual e histeroscopia de controle.

\section{Resultados}

As pacientes que foram submetidas à anestesia local suportaram bem o procedimento, queixando-se de cólicas do tipo menstrual de 
baixa e média intensidade durante os 8 minutos e 30 segundos de duração da ablação térmica. O seguimento variou de 6 a 18 meses. A avaliação da melhora foi padronizada da seguinte forma: as pacientes foram questionadas sobre a quantidade de fluxo menstrual antes do uso do balão classificando-o em: ausente/diminuído/ normal/inalterado. Utilizando os mesmos parâmetros, as pacientes foram interrogadas após o uso do balão. Os resultados são mostrados na Tabela 1.

Tabela1 - Resultados do questionário de avaliação da melhora da menorragia após o uso do balão térmico.

\begin{tabular}{|c|c|c|c|c|}
\hline \multicolumn{2}{|c|}{ Sintomas pré-tratamento balão } & \multicolumn{3}{|c|}{$\begin{array}{c}\text { Sintomas pós-balão } \\
\text { térmico }\end{array}$} \\
\hline & $\mathrm{n}$ & & $\mathrm{n}$ & $\%$ \\
\hline \multirow[t]{4}{*}{ Fluxo intenso } & 20 & Ausente & 2 & 10 \\
\hline & & Diminuído & 13 & 65 \\
\hline & & Normal & 3 & 15 \\
\hline & & Inalterado & 2 & 10 \\
\hline
\end{tabular}

Como sintomas adversos após a aplicação do balão, todas as pacientes queixaram-se de cólicas de leve a moderada intensidade nas horas que se seguiram à colocação do balão, havendo melhora com antiespasmódicos. Além de cólicas as pacientes referiram discreto sangramento nos dois primeiros dias, que foi seguido de secreção amarelada por mais de 2 semanas.

Duas das 20 pacientes mostraram-se insatisfeitas com o procedimento e 18 revelaramse satisfeitas com a melhora da sintomatologia.

A histeroscopia realizada logo após o procedimento revelou paredes e fundo uterino esbranquiçados.

Todas as pacientes foram submetidas a histeroscopia diagnóstica 40 dias e 6 meses após o procedimento. O achado histeroscópico após 6 meses foi de cavidade uterina reduzida, sinéquias no fundo uterino e algumas áreas de endométrio regenerativo.

Nas duas pacientes que não apresentaram melhora da sintomatologia, a histeroscopia revelou cavidade diminuída e endométrio em grande parte da parede posterior.

Não houve complicações como perfuração uterina, rotura do balão ou queimaduras do local.

\section{Discussão}

tratamento com balão térmico endo-uterino, 18 apresentaram melhora significativa da menorragia (90\%). Os resultados obtidos nesta série preliminar são semelhantes aos anteriormente encontrados com o uso da ablação histeroscópica do endométrio ${ }^{9}$.

A histeroscopia de controle realizada 40 dias após o procedimento teve como objetivo avaliar a destruição endometrial e realizar lise de sinéquias frouxas quando presentes junto ao orificio interno, para permitir a saída de fluxo menstrual assim como possibilitar novos exames histeroscópicos. A avaliação histeroscópica da cavidade uterina após 6 meses revelou os efeitos da queimadura do endométrio, caracterizada por destruição e formação de cicatriz.

Os resultados obtidos são semelhantes aos de outros centros europeus e americanos, que mostram uma melhora da menorragia em torno de $83 \%{ }^{14}$. O uso do balão térmico endo-uterino apresenta a vantagem de ser um procedimento de curta duração ( $8 \mathrm{~min}$ ), podendo ser efetuado em ambiente ambulatorial e compativel com rápida recuperação da paciente.

O uso do balão térmico endo-uterino para tratamento de menorragia com uso de anestesia local mostrou-se eficaz. Um número maior de casos, aliado ao acompanhamento mais longo, e estudos comparativos serão necessários para ratificar os resultados desta análise preliminar.

\section{SUMMARY}

Purpose: to evaluate thermal balloon endometrial ablation in the management of menorrhagia.

Study design: twenty patients were submitted to endometrial ablation using the thermal balloon device, between June 1996 and June 1997. Local anesthesia was used in 16 patients. The device was introduced into the uterine cavity. The duration of the procedure was 8 minutes and 30 seconds. Results: two patients (10\%) did not show improvement of the symptons. Eighteen patients (90\%) referred improvement of symptoms. There was no complication during and after the procedure.

Conclusions: The thermal balloon seems to be safe and efficient in the management of menorrhagia.

KEY WORDS: Uterine bleeding, abnormal. Endometrial ablation. Thermal balloon. Hysteroscopy.

Das 20 pacientes que foram submetidas ao 


\section{Referências}

1. Bayer SR, DeCherney AH. Clinical manifestations and treatment of dysfunctional uterine bleeding. JAMA 1993; 269:1823-8.

2. Snowden R, Christian B, editors. Patterns and perceptions of menstruation: a World Health Organization international collaborative study in Egypt, India, Indonesia, Jamaica, Mexico, Pakistan, Philippines, Republic of Korea, United Kingdom, and Yugoslavia. New York: St. Martin's Press; 1983.

3. Shaw RW. Assessment of medical treatments for menorrhagia. Br J Obstet Gynaecol 1994; 101 Suppl 11:15-8.

4. Nilsson L, Rybo G. Treatment of menorrhagia. Am J Obstet Gynecol 1971; 110:713-20.

5. Easterday CL, Grimes DA, Riggs JA. Hysterectomy in the United States. Obstet Gynecol 1983; 62:203-12.

6. Carlson KJ, Nichols DH, Schiff I. Indications for hysterectomy. N Engl J Med 1993; 328:856-60.

7. Goldrath MH, Fuller TA, Segal S. Laser photovaporization of endometrium for the treatment of menorrhagia. Am J Obstet Gynecol $1981 ; 140: 14-9$.
8. Loffer FD. Hysteroscopic endometrial ablation with $\mathrm{Nd}$ :Yag laser using a non-touch technique. Obstet Gynecol 1987; 69:679-82.

9. Deus JM, Coelho Junior ER, Martins JA, Lopes RGC, Viscomi FA. Ablação histeroscópica de endométrio: análise preliminar de 21 casos. Rev Bras Ginecol Obstet, 1994; 16:7-11.

10.DeCherney AH, Polan ML. Hysteroscopic management of intrauterine lesions and intractable uterine bleeding. Obstet Gynecol 1983; 61:392-7.

11.Vancaillie TG. Electrocoagulation of the endometrium with the ball-end resectoscope. Obstet Gynecol 1989; 74:425-7.

12.Droegemueller W, Greer B, Makowski E. Cryosurgery in patients with dysfunctional uterine bleeding. Obstet Gynecol 1971; 38:256-8.

13.Phipps JH, Lewis BV, Roberts T, et al. Treatment of functional menorrhagia by radiofrequencyinduced thermal endometrial ablation. Lancet 1990; 335:374-6.

14.Singer A, Almanza R, Gutierrez A, Haber G, Bolduc L, Neuwirth R. Preliminary clinical experience with a thermal balloon endometrial ablation method to treat menorrhagia. Obstet Gynecol 1994; 83:732-4. 